\title{
Image based Coin Recognition System - A Survey
}

\author{
Aby Sasi \\ PG student \\ Dept. of Computer Science \\ College of Engineering, Poonjar
}

\author{
Sreekumar K. \\ Assistant Professor \\ Dept. of Computer Science \\ College of Engineering, Poonjar
}

\begin{abstract}
Coins have very much importance in human's day to day life, which are used in everyone's daily routine like banks, super markets, vending machines etc: So, there is a basic need to automate the counting and sorting of coins. Coin recognition applications play an important role in industry and computer vision. The main objective of this system is to classify high volumes of coins with high accuracy and to recognize the coins of different denominations and count the total value of the coins. Many approaches developed for the coin detection and calculate its corresponding values. For this several approaches are compared here to improve the performance of the system
\end{abstract}

\section{General Terms}

Image Processing, Coin Recognition, Feature Extraction

\section{Keywords}

Image Analysis, Coin Recognition, Feature Matching, Rotation Invariant, Gradient Magnitude, RFR, Local Binary Pattern, Segmentation, Detection

\section{INTRODUCTION}

Coin recognition has been widely used in real life such as in vending machines, banks, supermarkets, charity organizations and ancient relics. Based on various recognition methods, three types of coin recognition systems are available in market: 1) Mechanical based 2) Electromagnetic based and 3) Image Processing based systems. Coins are differentiated with various patterns such as shape, size, surface design, weight etc. Coin Recognition is a difficult process because of its various rotations and widely change input patterns, noisy and cluttered images, which are the great challenges. Different algorithms used to detect, recognize and count coins, then produce their associated value. The mechanical method based systems use various parameters like diameter or radius, thickness, weight and magnetism of the coin to differentiate between the coins. But these parameters cannot be used to detect the different materials of the coins. It means that if we provide two coins-original and fake having same diameter, thickness, weight and magnetism but with different materials to mechanical method based coin recognition system then it will treat both the coins as original coin so these systems can be fooled easily. In the recent years coin recognition systems based on images. In these systems first, the coin image to be recognized is taken either by camera or by some scanning devices.After that these images are processed by using various image processing techniques like FFT, Gabor Wavelets, DCT, edge detection, segmentation, image subtraction, decision trees, ANN,SIFT etc .Based on various extracted features, different coins are recognized. Here we have a new approach to improve the performance of coin recognition system which is Rotation and Flipping Robust Region Binary Patterns using Gradient Magnitudes. RFR-GM was extracted from gradients magnitudes in coin images by local difference magnitude transform. RFR-GM provides much better accuracy compared to the original RFR. The comparative experiments showed that the RFR-GM approach had a better accuracy, faster feature extraction time, and smaller feature dimension. Therefore, RFR-GM is very suitable for image-based coin recognition.

\section{BASIC TERMS}

\subsection{RFR}

Rotation and flipping robust region binary pattern (RFR) has been proposed to detect rotated or flipped images. It uses region binary pattern template, which consists of several rings, and each ring is divided into several sub-regions. With the template, mean luminance of the sub-regions are computed and used to generate region binary pattern (RBP) features. RFR has high discrimination with a spatial structure as well as robustness for rotation.

\subsection{Gradient Magnitude}

The gradient magnitudes of a coin image are applied to extract

RFRs. The gradient magnitudes represent the structure of the coin image because they are computed from boundaries of characters and symbols.

\subsection{Feature Matching}

To compute the distance between two RFRs, we apply the distance matrix DM to compute distances between two RFRGMs. Let us suppose that Xq and Xr are RFR-GM index sets extracted from two coin images. Then, the distance between the two coin images can be computed as :

$\operatorname{dist}(\mathrm{Xq}, \mathrm{Xr})=\sum_{n-1}^{2 N-1} \mathrm{DM}(\mathrm{Xqn}, \mathrm{Xrm})$.

\section{RELATED WORKS}

\subsection{Coin Recognition System Using ANN}

Dr. Seema Bawa, Shatrughan Modi [1] developed a robust method for Automated Recognition System for Coins using Artificial Neural Network and is used for the recognition of Indian Coins with rotation invariance of various denominations such as '1', '2', '5' and '10'. For this, we have taken images from both sides. So this system is capable of recognizing coins from both sides. Features are extracted from images using various techniques such as Hough Transformation, Pattern Averaging etc. After passing the extracted features to a trained Neural Network., it has been achieved $97.74 \%$ recognition rate, which means only $2.26 \%$ miss recognition, which is quite encouraging.

\subsection{Saudi Riyal Coin Detection and Recognition}

Rawan S. Hassoubah, Amel F. Aljebry, Lamiaa A. Elrefaei [2] proposed a method to detect Saudi Riyal. In this paper a system is proposed, that accepts input images of Saudi riyal coins of the types quarter and half. Then, it recognizes Saudi Riyal coins through their radiuses. It starts by thresholding to 
produce binary image. Then, enhancing and detecting the edges. After that, using CHT to determine diameters of coins. Finally, recognize the coins and their associative value. It is applicable only to Saudi Riyal currency to differentiate between its two divisions half and quarter.

\subsection{Coin Recognition and Sum CountingSystem of Image Data Mining Using ANN}

Velu C M, P.Vivekanadan, Kashwan K R [3] presented an approach for Coin Recognition and Sum Counting System of Image Data Mining Using Artificial Neural Networks. The objective of this paper is to classify and recognize recently released Indian coins of different denominations, and count the total coin-value in terms of Indian National Rupees (INR). This system developed by combining Robert's edge detection method, Laplacian of Gaussian edge detection method, canny edge detection method and Multi-Level Counter Propagation Neural Network (ML-CPNN) based on the coins. The proposed method used for realizing a simple automatic coin recognition system more effectively. The Robert's edge detection method achieved $93 \%$ of accuracy and Laplacian of Gaussian method $95 \%$ of the result, the Canny edge detection method yields $97.25 \%$ result and the ML-CPNN approach yields $99.47 \%$ of recognition rate.

\subsection{Coin Recognition Using Local SpatialFeatures}

Unnikrishnan G, Sajith Sethu P [4] presented Automatic Coin Recognition Using Local Spatial Features. The proposed method uses the local features of the image for feature extraction. The method is invariant to rotation and translation and also the recognition is being done with single neutral image as train image. Normalized Local standard deviation filter is used for extracting the spatial intensity changes in the image. The key feature of this approach is that it uses single gallery image per coin for the recognition purpose and produces high recognition accuracy. Using background extraction of the coin and the decomposition of image into concentric circles increases its invariant property against rotation and translation. Even with this less number of train images an accuracy of $97.43 \%$ is being met. By adding more spatial features the accuracy can be increased hence the proposed method can be used as an efficient method for the face recognition system also.

\subsection{Coin Recognition System using ANN on Static Image Dataset}

Suchika Malik, Parveen Bajaj, Mukhwinder Kaur [5] presented a coin recognition and classification system based on new algorithms of Polar Fast Fourier Transform and image processing. This paper provides various coin recognition methods and as to get the best accuracy. Thereby, coins from more than 30 countries can be recognized and separated. Unknown coins are rejected. Further research will be carried out to improve the recognition performance and speed. These results are very encouraging

when considering the time costs with the neural network.

\subsection{CoinRecognitionSystemofImageSegme ntation by Heuristic Approach andHough Transform (HT)}

C.M.Velu and P.Vivekanandan [6] developed Indian Coin Recognition System of Image Segmentation by Heuristic Approach and Hough Transform (HT) based on coin table, which stores parameters of each coin. The proposed system by applying heuristic approach, based on the coin table yields $97 \%$ of accuracy in recognizing the coin image. The HT algorithm combining three features of HT algorithm a) Straight line detection b) Curve detection and c) Circle detection, we observe that the edge of the coin is recognized almost $100 \%$ of the coin image. Comparing to Sobel edge detection method the HT gives better results.

\subsection{Identification and Enumeration of Indian Coins Value UsingMorphological Operation}

Malatesh M, Smt. Anitha G [7], proposed the system to identify the coins in an image and calculates the total value of the coins which are on the image. There are several techniques involved such as image colour segmentation, edge enhancement, noise reduction, dilation, edge detection, Hough transform. The key to classify the coins based on its feature. Here radius and color are used. Once the radius is calculated, total value of the coins are calculated. MATLAB simulation is used to obtain results. Morphological operation shows positive sign for coin identification. Edge enhancement provides the clear edges of the coins to improve accuracy for coin detection. Also blob measurements provide better results.

\subsection{Ancient Coin Classification Using Reverse Motif Recognition}

Hafeez Anwar, Sebastian Zambanini, Martin Kampel, and Klaus Vondrovec [8], presented a system due to the lack of spatial information in the BoW(Bag of Visual Words) model and the requirements of the image based classification of ancient coins, we proposed a method for adding spatial information to the BoW model, which is invariant to scale changes, image rotations, and translation. This information is added using a three-step strategy that involves the automatic coin segmentation, application of a circular tiling scheme over the segmented image, and modeling the triangular geometric relationship of identical visual words in each tiling. It is shown that such a representation not only outperforms the BoW model but also was invariant to image rotations, scale changes, and translations. However, it was observed that the proposed method was more discriminating on smaller vocabulary sizes.

\subsection{An Efficient Coin Recognition Technique using Rotational Invariance Approach}

Nikita Shelgikar, Prof. L.M.R.J. Lobo, developed "Indian Coin Recognition with Rotation Invariance using Radial Blur Technique", [9] using rotation invariance approach, it is not necessary to place the coin at specific angle. Image Segmentation helps to reduces the amount of data required for processing. This system takes less time for processing and gives the best results.

\subsection{A Research - Develop An Efficient Algorithm To Recognize, Separate And Count Indian Coin From Image Using Matlab Processing}

Rathod Prahaladsinh Kanubha, Y.J.Parmar,Surendranagar[10] presents various systems developed and existing techniques of coin recognition based on image processing method for the better accuracy. It was shown that the described project contributes to the image based coin recognition and classifications. Thereby, coins from more than 31 countries 
can be recognised and separated from it. Further research will be carried out to improve the recognition result and also speed. And important thing is that, the Recognition time is very less.

\section{RESULT ANALYSIS}

This paper presents a comparative study of image based coin recognition. In the first approach "Coin Recognition System Using Artificial Neural Network", [1] fake coins are detected and it has been achieved $97.74 \%$ recognition rate. The second approach "Saudi Riyal Coin Detection and Recognition",[2] is applicable only to Saudi Riyal currency to differentiate between its two divisions half and quarter. The "Coin Recognition and Sum Counting System of Image Data Mining Using ANN"[3] method used for realizing a simple automatic coin recognition system more effectively. "Automatic Coin Recognition Using Local Spatial Features" [4] proposed the local features of the image for feature extraction. This method is invariant to rotation and translation. "Coin recognition and classification system based on new algorithms of Polar Fast Fourier Transform and image processing"[5] provides various coin recognition methods and as to get the best accuracy. Next approach "Indian Coin Recognition System of Image Segmentation by Heuristic Approach and Hough Transform" (HT) [6] based on coin table, which stores parameters of each coin. The proposed system by applying heuristic approach, based on the coin table yields $97 \%$ of accuracy in recognizing the coin image. In the seventh approach "Identification and Enumeration of Indian Coins Value Using Morphological Operation" [7] the edge enhancement technique provides the clear edges of the coins to improve accuracy for coin detection. Another approach "Ancient Coin Classification Using Reverse Motif Recognition" [8] shows the system which is invariant to scale changes, image rotations, and translation. The ninth approach "Indian Coin Recognition with Rotation Invariance using Radial Blur Technique", [9] using rotation invariance approach, it is not necessary to place the coin at specific angle. Also it helps to reduces the amount of data required for processing. "A Research - Develop An Efficient Algorithm to Recognize, Separate And Count Indian Coin From Image Using Matlab Processing”, [10] approach based on image processing method for the better accuracy. Here the Recognition time is very less.

Table 1.Comparison of Various ApproAches

\begin{tabular}{|c|c|c|c|c|c|}
\hline $\begin{array}{l}\text { SL } \\
\text { NO }\end{array}$ & METHOD & AUTHOR & $\begin{array}{l}\text { YEAR OF } \\
\text { PUBLICATION }\end{array}$ & ACCURACY & ADVANTAGES/DISADVANTAGES \\
\hline 1 & $\begin{array}{l}\text { Coin Recognition } \\
\text { System Using } \\
\text { Artificial } \\
\text { Neural Network }\end{array}$ & $\begin{array}{l}\text { Shatrughan Modi, } \\
\text { Dr.Seema Bawa }\end{array}$ & 2011 & 97.74 & Fake coins are detected \\
\hline 2 & $\begin{array}{l}\text { Saudi Riyal Coin } \\
\text { Detection and } \\
\text { Recognition }\end{array}$ & $\begin{array}{l}\text { Rawan S. } \\
\text { Hassoubah, } \\
\text { Amel F. Aljebry, } \\
\text { Lamiaa A. Elrefaei }\end{array}$ & 2013 & NA & $\begin{array}{l}\text { Input image with any } \\
\text { other type of currency is not applicable }\end{array}$ \\
\hline 3 & $\begin{array}{l}\text { Indian Coin } \\
\text { Recognition and } \\
\text { Sum Counting } \\
\text { System of Image } \\
\text { Data Mining } \\
\text { Using Artificial } \\
\text { Neural Networks }\end{array}$ & $\begin{array}{l}\text { Velu C M, } \\
\text { P.Vivekanadan, } \\
\text { Kashwan K R }\end{array}$ & 2011 & 99.47 & $\begin{array}{l}\text { Coin classification } \\
\text { technique is based on some assumptions } \\
\text { and computations }\end{array}$ \\
\hline 4 & $\begin{array}{l}\text { Automatic Coin } \\
\text { Recognition } \\
\text { Using } \\
\text { Local Spatial } \\
\text { Features }\end{array}$ & $\begin{array}{l}\text { Unnikrishnan G, } \\
\text { Sajith } \\
\text { Sethu P }\end{array}$ & 2013 & 97.43 & $\begin{array}{l}\text { It uses a single gallery } \\
\text { image per coin for the recognition. } \\
\text { Robust and high recognition } \\
\text { performance }\end{array}$ \\
\hline 5 & $\begin{array}{l}\text { Coin Recognition } \\
\text { System using } \\
\text { ANN on } \\
\text { Static Image } \\
\text { Dataset }\end{array}$ & $\begin{array}{l}\text { Suchika Malik, } \\
\text { Parveen Bajaj, } \\
\text { Mukhwinder Kaur }\end{array}$ & 2014 & 98.79 & $\begin{array}{l}\text { It is a low cost system. } \\
\text { Recognition time is very less. }\end{array}$ \\
\hline 6 & $\begin{array}{l}\text { Indian Coin } \\
\text { Recognition } \\
\text { System of Image } \\
\text { Segmentation by } \\
\text { Heuristic } \\
\text { Approach and } \\
\text { Hough Transform }\end{array}$ & $\begin{array}{l}\text { C.M.Velu } \\
\text { P.Vivekanandan }\end{array}$ & 2009 & 97 & $\begin{array}{l}\text { Zooming helps to make bigger size of a } \\
\text { coin image, by which recognition rate is } \\
\text { increased. }\end{array}$ \\
\hline 7 & $\begin{array}{l}\text { Identification and } \\
\text { Enumeration of } \\
\text { Indian Coins } \\
\text { Value Using } \\
\text { Morphological } \\
\text { Operation }\end{array}$ & $\begin{array}{l}\text { Malatesh M } \\
\text { Anitha } \mathrm{G}\end{array}$ & 2015 & NA & $\begin{array}{l}\text { Edge enhancement provides the clear } \\
\text { edges of the coins to improve accuracy } \\
\text { for coin detection. } \\
\text { Blob measurements provide better } \\
\text { results }\end{array}$ \\
\hline
\end{tabular}




\begin{tabular}{|c|c|c|c|c|c|}
\hline 8 & $\begin{array}{l}\text { Ancient Coin } \\
\text { Classification } \\
\text { Using Reverse } \\
\text { Motif } \\
\text { Recognition }\end{array}$ & $\begin{array}{l}\text { Hafeez Anwar, } \\
\text { Sebastian } \\
\text { Zambanini, Martin } \\
\text { Kampel, and } \\
\text { Klaus Vondrovec }\end{array}$ & 2015 & 82 & $\begin{array}{l}\text { Classifying ancient coins is a highly } \\
\text { complex task that requires years of } \\
\text { experience in the entire field of } \\
\text { numismatics. }\end{array}$ \\
\hline 9 & $\begin{array}{l}\text { An Efficient Coin } \\
\text { Recognition } \\
\text { Technique using } \\
\text { Rotational } \\
\text { Invariance } \\
\text { Approach }\end{array}$ & $\begin{array}{l}\text { Nikita Shelgikar, } \\
\text { Prof. L.M.R.J. } \\
\text { Lobo }\end{array}$ & 2014 & NA & $\begin{array}{l}\text { It is not necessary to place the coin at } \\
\text { specific angle. } \\
\text { Image Segmentation reduces the amount } \\
\text { of data required for processing. }\end{array}$ \\
\hline 10 & $\begin{array}{l}\text { To Recognize, } \\
\text { Separate And } \\
\text { Count Indian } \\
\text { Coins From } \\
\text { Image Using mat } \\
\text { lab Processing }\end{array}$ & $\begin{array}{l}\text { Rathod } \\
\text { Prahaladsinh } \\
\text { Kanubha, } \\
\text { Y.J.Parmar }\end{array}$ & 2014 & 96.5 & Recognition time is very less. \\
\hline
\end{tabular}

\section{CONCLUSION}

This paper focused on different systems for coin recognition based on image processing. Table 1 compared some researchers' work which is really helpful for study at a glance. There are so many methods has been proposed till now for the coin recognition, but still very less work has been done for better accuracy. The comparative experiments showed that each of these approaches improve better accuracy, smaller feature dimension and faster feature extraction time than the previous approaches had.

\section{REFERENCES}

[1] Dr. Seema Bawa, Shatrughan Modi Dept. of Computer Science and Engineering Thapar University Patiala 147004, India"Automated Coin Recognition System using ANN" ,International Journal of Computer Applications (0975-8887)Volume 26- No.4, July 2011

[2] Rawan S. Hassoubah (r.hassoubah@gmail.com), Amel F. Aljebry (a_jebr@yahoo.com) Lamiaa A Elrefaei (lamiaabdalah@yahoo.com), Faculties of Computing and Information Technology King Abdulaziz University Jeddah, Saudi Arabia "Saudi Riyal Coin Detection and Recognition" (proceedings of the 2013 IEEE Second International Conference On Image Information Processing)

[3] Velu C M1, P.Vivekanadan2, Kashwan K R3 ,1 R.S, Department of CSE, Anna University of Technology, Coimbatore 641 047, Tamil Nadu, India 2 Director, Knowledge Data Centre, Anna University, Chennai "IndianCoin Recognition and Sum Counting System of Image DataMining Using Artificial Neural Networks", International Journal of Advanced Science and Technology Vol 31, June,2011

[4] Unnikrishnan G, Sajith sethu P (PG Scholar, SCT College of Engineering, Trivandrum, Kerala, India (Assistant Professor , SCT College of Engineering, Trivandrum, Kerala,India "Automatic Coin Recogniton Using Local
[5] Spatial Features"IOSR Journal of VLSI and Signal Processing(IOSR-JVSP) Volume 3, Issue 5 (Nov. - Dec. 2013), PP 28-30 e-ISSN: 2319 - 4200, p-ISSN No. : 2319 - 4197www.iosrjournals.orgSuchika Malik, Parveen Bajaj, Mukhwinder Kaur ECE \&M.M U Sadopur Ambala India."Sample Coin Recognition System using Artificial Neural Network on Static Image Dataset", Volume 4, Issue 1, January 2014 ISSN: 2277 128X International Journal of Advanced Research in Computer Science and Software Engineering Research Paper Available online at: www.ijarcsse.com

[6] C.M.Velu, HOD of CSE, SKR Engineering College, Chennai - 602 103, India , P. Vivekanandan, Director, Knowledge Data Centre, Anna University, Chennai 600 025,India.email:cmvelu41@gmail.com,vivek@annauniv e.ed, "Indian Coin Recognition System of Image Segmentation by Heuristic Approach and Hough Transform (HT)" Int. J. Open Problems Compt. Math., Vol. 2,No.2,June2009

[7] Malatesh M Department of CS\&E,UBDTCE, VTU Karnataka, India, Smt. Anitha G, Department of CS\&E,UBDTCE, VTU Karnataka, India

[8] Hafeez Anwar, Sebastian Zambanini, Martin Kampel, and Klaus Vondrovec

[9] Nikita Shelgikar, Prof. L.M.R.J. Lobo, "Indian Coin Recognition with Rotation Invariance using Radial Blur Technique”, IJAIEM 2014 , 1M.E. (CSE) Department of Computer Science \& Engineering, Walchand Institute of technology, Solapur, India 2Associate Professor in Department of Computer Science \& Engineering, Walchand Institute of technology, Solapur, India

[10] Rathod Prahaladsinh Kanubha1, Y.J.Parmar2 , 1Student of M.tech E.C. in C.U.Shah Engineering College,Kothariya,Surendranagar. 2Assistnat Proffesor at C.U.Shah Engineering Collage,Kothariya,Surendranag. 